Capítulo 6

\title{
USO Y APROPIACIÓN DE TECNOLOGÍAS DE LA INFORMACIÓN Y DE LA COMUNICACIÓN. UNA APROXIMACIÓN A SU INVESTIGACIÓN EN LA ARGENTINA
}

\author{
Sebastián Benítez Larghi y Carolina Duek
}

\section{Presentación}

El análisis de las Tecnologías de la Información y de la Comunicación (TIC) y de la investigación sobre ellas, demanda una aproximación compleja porque su propia existencia en las sociedades contemporáneas es producto de diferentes tensiones y desarrollos de campos diversos. Desde las ciencias aplicadas, la ingeniería (en sus múltiples versiones), el diseño, hasta las ciencias sociales: todas las disciplinas convergen en un punto para ordenar la manera en la que las nuevas tecnologías se conciben, diseñan, presentan y (en los mejores casos) circulan y adoptan por parte de los consumidores y receptores. Fidler (1998) identifica estos procesos con una noción interesante, la mediamorfosis, que consiste en concebir la evolución de los dispositivos en el proceso histórico en el que se insertan pero, a la vez, identifica la aparición de los nuevos soportes y dispositivos como parte de una co-evolución.

Para Fidler, hay una coexistencia y una convivencia entre nuevas y viejas maneras de comunicarse y las nuevas invenciones no suponen, necesariamente, reemplazos sino que forman parte de un encadenamiento del pasado en las invenciones presentes. La aparición de la televisión generó muchos pronósticos terribles para la radio: desde su desaparición hasta su inutilidad. Hoy, muchísimas décadas después, podemos identificar la coexistencia entre ambos medios y soportes pero, a la vez, su evolución en lo que respecta a la calidad de la transmisión y de contenidos (Flichy, 2013). Es por ello que analizar los procesos en términos de mediamorfosis es abordar todas las formas de comunicación del pasado y del presente y construir conjeturas e hipótesis sobre el futuro de los dispositivos, de los soportes, de los usos y de las apropiaciones. "Si mira- 
mos al sistema de medios de comunicación como un todo, veremos que los nuevos medios no surgen por generación espontánea ni independientemente" (Fidler, 1998: 57).

Wiliams (1974) sostiene que es necesario restaurar la intención en el proceso de investigación y desarrollo de las nuevas tecnologías. $Y$ es por ello que identifica una necesidad (que no necesariamente es explícita) a la que las invenciones y avances dan una respuesta. En este sentido, la tecnología es "buscada" y desarrollada con objetivos específicos y prácticas que están previamente concebidas (si bien luego pueden cambiar, distorsionarse y hasta ser contrarias a lo imaginado).

Varela (2005), en su investigación sobre la televisión, identifica diferentes elementos que formaron parte del imaginario social en el momento de la aparición de la televisión (que periodiza en diferentes etapas, véanse pp. 15 y ss.) y sostiene que la apropiación de la televisión por parte de la sociedad demoró casi veinte años. El miedo, la desconfianza y lo oneroso que era adquirir estos bienes, los ubicaban en un terreno de la duda y de temores. Y aquí reaparecen los argumentos de Fidler sobre mediamorfosis: la irrupción de nuevos dispositivos, soportes y medios de comunicación atraviesan diferentes etapas necesarias hasta su expansión, consolidación y final naturalización (cristalizada en expresiones como "no podría ya vivir sin mi celular", por reconstruir uno de los tantos ejemplos posibles).

Gubern (2000) describe los hogares (de clase media, con acceso a los dispositivos y a la conectividad) como "cuevas aterciopeladas" desde las que se puede participar, trabajar, comprar e interactuar con el mundo exterior gracias a las nuevas Tecnologías de la Información y de la Comunicación. Estas cuevas serían la culminación de los procesos que estamos comenzando a presentar: la informatización llevada a sus extremos (imaginemos la imposible conectividad global total y el acceso igualitario y democrático a las pragmáticas de uso) y la modificación (¿irreversible? ¿̨revolucionaria? ¿magnífica?) de las formas de comunicación entre las personas.

Co-existencia, convivencia, intención, apropiación, "cuevas aterciopeladas": cinco palabras clave que nos introducen al universo sobre el cual se proyecta y despliega este capítulo. Indagar las condiciones de producción de las investigaciones es siempre la clave de acceso para pensar y reflexionar sobre las TIC. Las preguntas, las hipótesis y los arcos conceptuales que vamos a recorrer son aquellos que relevamos a lo largo de nuestra tarea de 
(meta) investigación orientada al consumo, al uso y a la apropiación de las TIC.

\section{Objetivos e hipótesis: acerca de la investigación}

El objetivo del presente capítulo es abordar la investigación realizada sobre las prácticas, las apropiaciones y el consumo de dispositivos y plataformas vinculadas con las TIC. La investigación local sobre este sub-campo -aún en desarrollo dentro del campo de estudio sobre los consumos culturaleses, a la vez, amplia y acotada.

En este sentido, diseñamos tres hipótesis con las que trabajamos a lo largo del capítulo como manera de construir una aproximación productiva al corpus de trabajo. En primer lugar, sostenemos que la investigación sobre TIC no incluye en sus libros, capítulos, artículos y ponencias una reflexión sobre la caducidad de los temas, objetos y conceptos abordados. Los cambios, los nuevos dispositivos y plataformas de contacto modifican de manera constante y sostenida las prácticas de recepción y, a su vez, vuelven caducas conclusiones de investigaciones científicas que son vigentes por períodos cada vez más acotados. Esta dimensión, sostenemos como hipótesis, no aparece como elemento de las reflexiones del corpus.

En segundo lugar, sostenemos que la investigación sobre uso de redes sociales y espacios vinculados con las TIC aparece con frecuencia en el corpus dada la facilidad de acceso a las interacciones y publicaciones de los usuarios. De este modo, las limitaciones de financiamiento para realizar investigaciones a largo y mediano plazo se desplazan hacia "análisis de casos" que son acotados y accesibles por el mero acceso a los dispositivos (y al contacto con los informantes potenciales y/o con sus cuentas activas en redes sociales, plataformas y demás dispositivos).

En tercer lugar, la investigación sobre uso, apropiación y consumo de TIC no solo es sencilla de realizarse por la accesibilidad a los informantes sino que, en los casos de análisis de redes sociales, plataformas on line y demás posibilidades, las competencias necesarias para el manejo de las acciones en dichos espacios son verdaderamente sencillas y no precisan de alfabetizaciones especiales ni de pragmáticas de uso para iniciarse. Es por ello que estos espacios de interacción y de conexión con otros con baja 
demanda en lo que respecta a las habilidades aparecería como "competencia desleal" con las demandas de las escuelas y de los diseños curriculares que sí (y muy lógicamente) demandan del niño y joven alumno una serie de destrezas y aprendizajes escalonados y articulados.

Será a través de estas tres hipótesis que presentaremos los resultados de la investigación sobre las publicaciones académicas sobre TIC, usos, apropiaciones y consumo. El capítulo está organizado en diferentes apartados para ordenar la argumentación: acerca de la construcción de los objetos de estudio; formatos y sistematizaciones; palabras clave y, finalmente, abordajes y estrategias metodológicas.

Estos ejes son los que nos permitirán presentar los resultados articulando en cada eje y en cada análisis una mirada transversal sobre el corpus para poder extraer conclusiones que, si bien no serán definitivas, sí nos van a permitir identificar las principales formas de hacer investigación sobre uso, apropiación y consumo de TIC en la Argentina contemporánea.

\section{Acerca de la construcción de los objetos de estudio}

La aparición de las TIC ha suscitado un enorme interés dentro de las ciencias sociales contemporáneas. En este sentido, existe un consenso acerca de que asistimos a una revolución tecnológica casi sin precedentes. Es por ello que, a medida que la computadora, Internet y el teléfono celular se fueron sumando a nuestra vida cotidiana, la inserción de estas tecnologías en el ámbito del trabajo, el consumo, la cultura y la política ha constituido el objeto de estudio de numerosos especialistas. Desde diversos ángulos se observa la relación de las TIC y los procesos de producción y circulación de mercancías, los modelos de trabajo y los hábitos de ocio, las formas de comunicación interpersonal y los mecanismos de participación y representación política. La preocupación aparece puesta entonces en los posibles cambios generados en torno a las nuevas tecnologías en las distintas esferas de la vida social. Esta temática ha tenido un desarrollo exponencial en los últimos años. Distintas disciplinas sociales han abordado el estudio de las TIC privilegiando diferentes dimensiones de análisis. Sin pretensiones de ser exhaustivos -que, dada la vastedad del universo, sería imposible- en líneas generales podemos resumir estos abordajes en torno a los 
siguientes tópicos: la interrelación entre el surgimiento de las nuevas tecnologías, las transformaciones societales contemporáneas y la conformación de la llamada "sociedad de la información"; la relación TIC, infancias y juventud(es) con especial interés en la producción de subjetividades, la sociabilidad y la dimensión de lo lúdico; la semiosis propia de los nuevas tecnologías y el estudio de nuevos lenguajes y formas de escritura.

Dentro de esta amplitud, y sin desconocer la riqueza y pertinencia de cada una de las dimensiones, el presente capítulo se propone reconstruir el estado de la cuestión de aquellas investigaciones sociales dedicadas a estudiar los procesos de uso y apropiación de las TIC en la Argentina contemporánea.

En relación a la construcción del objeto de estudio, destacamos tres elementos centrales característicos del corpus analizado: a) los fenómenos abordados y su devenir cronológico; b) la problematización teórica del vínculo entre lo social y lo tecnológico; c) los modos de abordaje privilegiados.

Si bien ya desde sus comienzos, los estudios de las TIC nunca ignoraron el rol activo de los usuarios (dado que su manipulación resultaba evidente), los análisis se limitaron, en un primer momento, a las interacciones en el ciberespacio como experiencias paralelas al mundo real obviando la influencia de las dimensiones de la vida cotidiana presentes en todo proceso de apropiación. Luego, desde comienzos del siglo, esta tendencia comenzó a ser revertida otorgando más peso a la contextualización y a las conexiones entre el mundo on line y el mundo off line. Así, los trabajos que conforman el corpus analizado comparten esta nueva perspectiva. Sin embargo, se observa que los fenómenos abordados por los trabajos del corpus se fueron desplazando a lo largo del período abarcado (2000-2012). Mientras que en los primeros años del siglo persistía una fuerte preocupación por la cuestión del acceso a la tecnología y sus vínculos con las desigualdades sociales -cuestión que se pone de manifiesto en la apelación recurrente a la noción de brecha(s) digital(es) - (Bianchi, López y Perera, 2008; Marchetti y otros, 2008; Monje, s/d, entre otros); a medida que el acceso a la computadora, la telefonía celular y las conexiones a Internet fue masificándose, el foco de atención pasó a concentrarse en los usos de estos artefactos: primero en la aparición de los blogs (Sandoval, 2008) y, más específicamente hacia el final de período, en el fenómeno de las redes sociales (Remondino, 2012; Arabito, 2011; De la 
Torre y Vaillard, 2012, son algunos ejemplos) y en las nuevas formas de producción de contenido (Jacobo, 2011). Así, podemos afirmar que este desplazamiento de los accesos a los usos y apropiaciones nos habla de una construcción del objeto de estudio donde la investigación persigue el devenir del propio movimiento de los artefactos, plataformas y usos.

En cuanto al modo de problematización del vínculo entre tecnología y sociedad, podemos afirmar que, a pesar de partir de una amplia variedad de propuestas, abordajes y corpus teóricos, todos los estudios analizados tienen como denominador común una fuerte preocupación respecto a qué hacen los sujetos con/frente/a través de las TIC. En este punto se observa una inquietud común, a veces más explícita y otras implícitamente, por no caer en ningún tipo de reduccionismo, ni tecnológico ni sociológico. Podríamos decir que a nivel teórico se intenta evitar posiciones tanto apocalípticas como integradas respecto al potencial transformador de las TIC pero al mismo tiempo existe un lineamiento ideológico que prevalece: en líneas generales la concepción de la tecnología se construye desde una perspectiva socio-técnica. Este balance permanente se manifiesta en el uso problematizado de la noción de impacto como punto de equilibrio que matiza tanto posturas que consideran a la tecnología como el locus del cambio social como relativismos sociológicos que otorgan plena omnipotencia a los usuarios. Es decir, aparece como idea fuerza de los trabajos la problematización de la categoría de impacto alejándose de la noción funcionalista de efectos pero también de la noción del receptor multi-acentuado propio de la etnografía de audiencias.

El texto de Marchetti y otros (2008) es un buen ejemplo. Si bien allí los autores construyen un problema de estudio poco frecuente dentro de la literatura sobre el tema como los vínculos entre discapacidades, TIC y desigualdades, el marco teórico que le da sustento ilustra de manera cabal el balance entre posiciones apocalípticas e integradas en la medida que el uso y la fusión de medios dependen de las capacidades de los sujetos para domesticar los artefactos. "En este marco, el sujeto es un sujeto de cambio que trastoca, modifica todo aquello que le es presentado y por lo tanto hace necesario remitirse a las prácticas individuales y a la acción creadora de quienes deciden exponerse ante los dispositivos de comunicación" (Marchetti y otros, 2008: 3) Así, la perspectiva del actor aparece recurrentemente recuperada como preocupación central y, por ende, relativizando 
el argumento que posiciona a las TIC como objetos fetichizados capaces de reducir o superar las desigualdades por sus cualidades intrínsecas. El foco está puesto entonces en las mediaciones (económicas, sociales, culturales, simbólicas) que constituyen los procesos de apropiación. Las categorías de pantalla, interfaz y dispositivo dan cuenta de estos procesos, por cuanto se trata de "categorías que nos permiten acercarnos a esta complejidad e imbricación entre producción y consumo, en un entorno digitalizado. [...] la interfaz activa la inmediatez, y la pantalla es lo que la soporta; la interfaz es un 'entre dos' que define el tipo de relación que se establece con el usuario, reconstruyendo y alterando al mismo tiempo las dos identidades (hombre-máquina)" (Marchetti y otros, 2008: 1). Desde esta perspectiva, algunas discapacidades producen una alteración y hasta la pérdida de algún sentido o función. Esto genera el reemplazo o máximo desarrollo de otras funciones o sentidos con la consecuente adquisición de otras habilidades y destrezas. A partir del uso de las TIC, las personas con discapacidad extienden alguno de los sentidos o funciones carentes, suplantan su ausencia, y de esta manera puede entenderse una alteración de la proporción sensorial. La ausencia, la carencia, disminuye sus efectos al entender a las tecnologías como prótesis del propio cuerpo. El teléfono móvil, por ejemplo, "devuelve" a los sordos la capacidad perdida de comunicarse de igual modo que las personas que oyen a través de un "lenguaje común" como es el SMS. De este modo, "las tecnologías parecen tener una función social-humana para la cual no fueron en principio pensadas, que excede el mero hecho de estar comunicados, adquiriendo un significado mucho más rico" (Marchetti y otros, 2008: 6). Por lo tanto, existe una divergencia tecnológica tanto desde el punto de vista de la producción como, consecuentemente, de la recepción. En este marco en el que los dispositivos y soportes tecnológicos se han multiplicado y perfeccionado en un proceso de convergencia generando audiencias divergentes, la discapacidad empieza a entenderse como una cuestión de divergencias, de diversidad de necesidades y no como una cuestión de limitación personal.

La cuestión de la divergencia entre producción y recepción da cuenta del margen de interpretación que todo proceso de codificación habilita. Ya decía Hall en su famoso artículo "Codificar/Decodificar", "los códigos de codificación y decodificación pueden no ser perfectamente simétricos" (Hall, 1979: 3). Allí se despliega un campo de interpretación con un am- 
plio abanico de posibilidades de "lecturas" (ya sea de mensajes simbólicos como de los significados otorgados a los artefactos). Estas posibilidades van desde el reconocimiento pleno y la identificación con las intenciones del emisor/diseñador hasta la resistencia, pasando por diversas instancias de negociación que devienen en un uso divergente o tergiversador. El posicionamiento teórico de los trabajos del corpus y la marcada preocupación por el equilibrio ponen de manifiesto que estas posibilidades no son infinitas. Es decir, el usuario no puede ejercer una lectura con plena autonomía sino que esta es relativa por cuanto todo proceso de codificación implica, al mismo tiempo, la intención de imponer un patrón de lecturas preferente. "Cualquier sociedad o cultura tiende, con diferentes grados de clausura, a imponer sus clasificaciones del mundo político, social y cultural. Estas constituyen el orden cultural dominante aunque no sea unívoco o no contestado" (Hall, 1979: 6).

Finalmente, cabe hacer dos señalamientos respecto a los modos de abordaje elegidos en la construcción de los objetos de estudio como marca característica de este sub-campo de los consumos culturales dedicado a los usos y apropiaciones de las TIC. Por un lado, se observa una tensión entre la descripción de la novedad de los objetos y la necesidad de sistematizar lo existente para abordarlos. Cuestión que nos habla de un sub-campo aún no constituido, que permanentemente oscila entre la actualización frente al vertiginoso devenir de los fenómenos abordados y la revisión de los conocimientos acumulados. De allí que cada una determinada cantidad de trabajos sostenidos en trabajos empíricos aparezcan esfuerzos de sistematización de los hallazgos. Se trata de esfuerzos que se canalizan ya sea por vía de la reconstrucción de estados de la cuestión como por vía de trabajos eminentemente teóricos que, muchas veces, adopta un formato ensayístico, cuestión que también da cuenta del estado en proceso de consolidación del sub-campo de estudio. Por otro lado, debe señalarse otra invariante cultural de la investigación de este sub-campo: se trata de investigaciones que por lo general resultan muy fechadas. Es decir, la mayoría de los estudios construyen sus objetos muy atados a modalidades de accesos, programas o plataformas relevantes al momento de realizar la investigación pero que sus conclusiones quedan desactualizados tan solo en un par de años. De esta forma, continuamente se camina al filo de la caducidad. ¿Qué nos permiten pensar 
hoy las investigaciones realizadas en los ya prácticamente extintos cibercafés? ¿Cuán válidos serán los hallazgos sobre los modos de sociabilidad en redes sociales virtuales como Facebook o Twitter cuando estas ya no ocupen el centro de la escena? En este sentido, podríamos concluir que esta caducidad no hiere la legitimidad de la investigación pero sí la de sus resultados.

\section{Formatos y sistematizaciones para constituir el sub-campo}

Uno de los datos interesantes que se desprenden del corpus (que, reiteramos, no contiene la totalidad del universo de las investigaciones existentes) es la distribución de los formatos de publicación: los libros completos sobre el tema son excepcionales mientras que las ponencias y presentaciones a congresos y jornadas y los capítulos tienden a ser los modos preferenciales de publicación. Por supuesto que esta dinámica se vincula con la ya mencionada caducidad de los temas de investigación y, también, con la necesidad de presentar sistematizaciones que abran el sub-campo de investigación como tal.

El libro de Finquelievich y Prince (2007) titulado El (involuntario) rol social de los cibercafés es una de las excepciones que encontramos junto con Los usos de las Tics. Una mirada multidimensional, de Quintar, Calello y Aprea (también de 2007) y, del mismo equipo, el libro compilado por Roxana Cabello titulado Yo con la computadora no tengo nada que ver. Un estudio de las relaciones entre los maestros y las tecnologias informáticas en la enseñanza de 2006. Estos tres libros nos invitan a la reflexión sobre las características de los objetos de estudio abordados y, también, nos convocan a identificar los intentos de sistematización bibliográfica e investigativa existentes. No es casual que entre 2006 y 2007 encontremos estas tres publicaciones: hay una necesidad creciente de organizar el sub-campo, de delimitar sus alcances, preocupaciones y dinámicas.

Dijimos más arriba que la caducidad de los objetos de estudio se vinculaba con un tiempo de avances y de presentación de nuevos dispositivos en sociedad pero que eso no le quitaba legitimidad (pragmática ni procedimental) a las investigaciones publicadas. En síntesis, relevamos una preocupación por sistematizar, organizar y delimitar un área temática en tanto un sub-campo. 
El cuarto libro que encontramos en la constitución del corpus para este capítulo es de 2008 y se titula Ciberculturas juveniles, editado por Marcelo Urresti. El libro comienza con la presentación de las preguntas de la investigación colectiva que dio pie a la existencia del libro: "[...] el ciclo posterior de indagaciones comenzó con una lectura profunda del estado del arte en la materia, actividad que mostró rápidamente el vasto terreno por recorrer, pleno de promesas e incógnitas que reclamaban intervención y esclarecimiento" (Urresti, 2008: 8). En el comienzo nos encontramos con el problema ya mencionado: la definición de un sub-campo dentro de las ciencias sociales que releve las preocupaciones, investigaciones y nociones centrales para abordar la relación de (en este caso) los jóvenes con las TIC en diferentes contextos de uso y apropiación.

Urresti presenta, a continuación, un capítulo en el que se propone sintetizar los textos centrales y las nociones fundacionales del sub-campo en el que se asienta la investigación: ciberculturas juveniles, vida cotidiana, subjetividad, pertenencia y TIC. "Es entonces en el contexto de una sociedad massmediatizada que las nuevas tecnologías de la comunicación y la información encuentran el terreno propicio para desarrollarse" (ibídem: 21). La noción de ruptura en relación con un estado social anterior y las formas de vinculación son elementos que recorren todo el libro (con especial énfasis en la primera y en la segunda sección). Los usos, las apropiaciones se organizan según diferentes dimensiones de análisis que selecciona el equipo de trabajo: el uso de Internet por parte de jóvenes de clase alta (Pahor, 2008), la relación entre Internet y los jóvenes de clase media (Fernández Jeansalle, 2008) y el texto de Bouille (2008) sobre los cibercafés y los jóvenes de sectores populares urbanos. Estos tres trabajos analizan los diferentes usos y relaciones posibles en y a través de las TIC.

Los jóvenes entrevistados por Bouille sostiene que usan Internet para acceder "más rápida y fácilmente a la información para la escuela, poder prescindir de los libros que tienen un costo mayor, comunicarse de manera más económica que la que ofrece el teléfono, poder conseguir artículos (como los celulares) más baratos y acceder a cierta información específica particular (por ejemplo, manuales de usos)" (Bouille, 2008: 120). Aquí aparece como elemento clave la ausencia, en los años de investigación previos a la publicación, de las redes sociales como Facebook que, según estudios actuales, ocupa una inmensa mayoría del tiempo, de los 
usos y de las apropiaciones de los jóvenes de todas las clases sociales en relación con las TIC -véase por ejemplo la Encuesta Nacional de Consumos Culturales (2014) para precisar estos datos-.

La segunda sección del libro se ocupa de los blogs y de los fotologs (Goszczynsky, 2008), del software libre (y la relación con los hackers) (Vannini, 2008) y de los llamados webmasters (productores y diseñadores de páginas web) (Bonacci, 2008). Todos estos temas, si bien relevantes, despliegan las limitaciones respecto de la vigencia de los dispositivos y temas: el avance de las redes sociales en la definición de los usos y de las plataformas de interacción ha sido a la vez abrupto y exponencial dejando el espacio de los blogs, fotologs y de los webmasters en un lugar desplazado de la centralidad de la escena. No decimos que hayan desaparecido del sub-campo sino que ocupan una segunda posición, una segunda línea luego de las plataformas multimediales de las redes sociales como Facebook, Twitter, Instagram, entre otras.

Probablemente, en no mucho tiempo, este texto que escribimos tenga las mismas dificultades que observamos, ya no en Urresti (2008) sino en la totalidad de la bibliografía que compone este corpus. Pareciera que los tiempos académicos están seriamente desacompasados a los de los dispositivos o, en otra hipótesis, simplemente no hay forma de seguir el ritmo industrial/comercial de presentación de novedades (o pseudo-novedades) por parte del mercado de bienes y servicios. Encontramos una amplia variedad de textos sobre las TIC que no abordan la recepción que están vinculados con preocupaciones de la economía política: la colección "Futuribles" de editorial La Crujía, por ejemplo, Becerra (2003) [Sociedad de la Información: proyecto, convergencia, divergencia], Mastrini, Loreti y Baranchuk (2007) [Participación y democracia en la sociedad de la información], solo por mencionar algunos de los investigadores (y equipos) destacados sobre el tema.

El abordaje del corpus en relación con los formatos, temas y formas de publicación nos devela, en esta aproximación, un universo complejo de investigaciones en el que encontramos un desajuste de los tiempos del mercado y los de producción académica pero, también (y complementariamente), una necesidad constante de sistematizar y organizar la bibliografía existente en estados de la cuestión que permitan visibilizar el subcampo de manera clara (y provisoria). 


\section{Palabras clave}

La inmensa variedad de opciones a la hora de identificar tres o cuatro palabras clave para ubicar un texto en un área de investigación hace difícil la organización de muchos textos de un corpus a la luz de estas palabras seleccionadas. En muchos textos del corpus no estaban consignadas las palabras clave y tuvimos que intervenir definiéndolas para poder procesar todos los textos en función de las mismas categorías.

En el camino de síntesis y de análisis encontramos una manera de organizar los textos del corpus en cuatro grandes ejes que se ordenan en función de palabras clave:
a. Uso/apropiación
b. Lectura
c. TIC/Dispositivos
d. Redes sociales

Será a través del abordaje de estas cuatro palabras clave que trabajaremos, en este apartado, el corpus seleccionado de autores y de investigaciones.

\section{Usos/Apropiaciones}

La presencia de la preocupación por los usos y por las apropiaciones que diferentes grupos, subgrupos, (segmentos de) clases sociales y demás agrupaciones posibles, ocupa una inmensa parte del corpus con el que trabajamos. Abordar la relación entre los sujetos sociales y las TIC alejados de una perspectiva determinista, abre un universo productivo de investigación pero, a la vez, demanda precisiones teórico-conceptuales que cada texto retoma y define.

En el texto de Benítez Larghi et al. (2012) se define como objetivo: "explorar de qué modo los jóvenes de sectores populares urbanos se apropian de las TIC en un contexto de acceso público compartido, y de qué manera condicionan sus usos tecnológicos los diferentes tipos de espacios que ofrecen acceso público" (2012: 11). Encontramos, en esta cita, la definición del objetivo de investigación (modos de apropiación/condicionamientos de uso) y de un contexto específico (acceso público compartido). 
No encontramos textos que no se planteen la cuestión del acceso y la definición específica de un contexto y ejes de análisis. Y ese no es un elemento menor a la hora de analizar el corpus.

Por otra parte, en el texto de Alzina, Benyo y Durán Prieto (2011), se plantean el acceso a las TIC como forma de hacer frente a la "brecha digital" y, a la vez, contribuir a la formación de opinión y a la democratización en el marco de asambleas barriales: "hay un reconocimiento por parte de estos actores de que existe algún tipo de limitación por estar excluidos de las TIC [...] sostenemos por ello que el verdadero impacto de las TIC se ha producido en el plano de las expectativas de los integrantes de estas organizaciones barriales que han expresado su deseo de usar las nuevas tecnologías para difundir sus actividades, y sus pensamientos" (2011: 15). Respecto de los usos y de las expectativas encontramos en este texto un desajuste: por un lado pareciera que "quedarse afuera" se vincula con la imposibilidad de acceder a las TIC pero, por otro, hay una conciencia de que el acceso no garantiza igualdad frente a otros (aunque sí una posibilidad de contar con herramientas para la formación de opinión sobre temas cercanos).

Continuando la preocupación por la "brecha digital”, Paz (2012) aborda el uso de las TIC con fines educativos: "la brecha digital no es sólo una brecha que se da entre distintos estratos sociales o generacionales, sino que existe una brecha constatable a través del análisis cualitativo, que atañe al contraste entre la enseñanza escolar y otros ámbitos de socialización (2012: 2). Identifica, luego, la forma de uso de la computadora en el aula: 'en cuanto al 'proceso de búsqueda', que se dice promover, no hay en realidad un efectivo proceso de búsqueda, lo que demanda lectura, contrastación y análisis de la información disponible. Generalmente se quedan con la primera página que encuentran, la que generalmente es Wikipedia" (2012: 9). La falta de análisis y de jerarquización de la información aparece como la principal característica en la definición de la apropiación de las TIC en el aula.

Finalmente, en la relación entre usos, apropiaciones y TIC aparecen "[...] percepciones sobre la computadora e Internet, y los efectos que tiene la relación con esta tecnología en términos de construcción de habilidades, acceso (y permanencia) en el sistema educativo, empleabilidad, sociabilidad, compromiso y participación en la vida pública" (Benitez Larghi et al., 2012: 11). Esta definición de Benítez Larghi nos permite 
cerrar esta primera palabra clave: no importa en qué grupo ni en qué contexto se lleve a cabo la investigación; lo que aparece sostenidamente es la relación entre las percepciones de las TIC, las expectativas y las pragmáticas de uso vinculadas con la sociabilidad y la participación pública.

\section{Lectura}

Resultó interesante identificar las formas en que la lectura aparecía como una gran preocupación en el corpus. Las transformaciones de la lectura y las nuevas formas que adquiere a la luz de las TIC son los dos ejes que encontramos con más recurrencia en los textos seleccionados.

Albarello sostiene que "la pantalla de la computadora y el dispositivo impreso invitan a dos modos de lectura diferentes, aunque no opuestos, sino que, en muchos casos, son complementarios. [...] La pantalla invita a mantener explícitas las condiciones de lectura por su naturaleza metamedium que habilita la posibilidad de hacer varias cosas a la vez" (2001: 205). La simultaneidad y la complementariedad aparecen como dos claves para entender la lectura en la actualidad: no se trata de sustituciones sino de complementariedades. Hoy, según Albarello, se puede leer en la pantalla y de manera simultánea diferentes textos que demandan variables grados de atención. Esta postura (si bien discutible) habilita una visión no apocalíptica en lo que concierne a la lectura en la actualidad. No se trata de analizar la lectura como una práctica del pasado sino de encontrar en ella los rasgos propios de los tiempos contemporáneos.

Maestri (2011), en la misma línea, al definir a las audiencias digitales sostiene que: "la comunicación provoca la conjunción de medios de comunicación y tecnologías de la comunicación e información dando lugar a la convergencia de medios. [...] la implicancia entre diferentes medios de comunicación y a los cambios culturales que se derivan de la interactividad entre los sujetos" (2012: 30). La convergencia es abordada aquí como un rasgo de la actualidad que debe ser comprendido como uno de los elementos clave para la reconfiguración de las prácticas. Los nuevos soportes y dispositivos habilitan nuevas formas de vinculación y de caracterización de las audiencias en tanto lectoras y productoras de mensajes. 
Cornejo y Tapia (2011) cierran esta entrada caracterizando las relaciones interpersonales actuales y detallando las modificaciones que se han dado a la luz de las TIC en lo que respecta a la relación con las aplicaciones y soportes y, también, con las formas de lectura y de recepción.

La lectura es una preocupación clave que se releva en la bibliografía y se vincula con la historia misma del campo de la comunicación y la cultura y con las herramientas metodológicas que se han utilizado (y que se utilizan) para desarrollar investigaciones sobre las TIC. Leer, producir, escribir e interactuar aparecen como cuatro dimensiones clave de la vida cotidiana de las audiencias digitales, tal como las define Maestri (2011).

\section{TIC/Dispositivos}

La interacción con y a través de los dispositivos aparece, como dijimos, como una gran preocupación del sub-campo. No se trata solo de analizar qué hacen las audiencias con los dispositivos sino identificar las formas en las que se construyen entramados (inestables) a través de los soportes que habilitan las TIC. Es por ello que la tercera palabra clave la conforman las TIC/Dispositivos en tanto variables fuertemente intervinientes en las prácticas de producción, apropiación, consumo, uso y recepción.

Andrés (2012) contrapone la noción de interactividad con la de "interpasividad" y sostiene que: "la actividad del internauta sería una falsa actividad, ya que en realidad es un aparato el que realiza la actividad de mirar, navegar e, incluso, gozar. Desde esta perspectiva, la computadora o el teléfono móvil son los que actúan y, por lo tanto, la participación no sería tal: todo acontece como si el usuario fuese activo al intervenir en las redes y producir contenidos, pero en la mayoría de los casos solamente se encuentra delante de un aparato seleccionando opciones y navegando de un sitio a otro" (Andrés, 2012: 106).

De manera muy polémica, Andrés plantea una diferenciación en la que aparece "subjetivado" el dispositivo y el sujeto deviene mero operador de una plataforma que desconoce. Es interesante pensar la manera en la que este sub-campo demanda constantemente la re-evaluación y la redefinición de las nociones y conceptos clave para interpretar la relación entre los usuarios y los dispositivos. No se trata de desplazar el goce del sujeto 
(que, sostenemos, existe de variadas formas) sino que las características propias de la relación de estos con las TIC está organizada, claramente, en función de los dispositivos, ya no como meros mediadores sino como variables intervinientes de las prácticas y de las acciones posibles (e imposibles) por parte de los usuarios/consumidores (para ampliar esta dimensión consultar Aón y Zapata, 2011).

Moscoloni y Castro Rojas (2011) reconocen que ya no es posible analizar las transformaciones tecnológicas como mero instrumento de cambio cuantitativo, sino que es importante poner en cuestión el uso y los consumos, en los contextos sociales en los que estos se inscriben. La inclusión de los contextos contiene, en todos los casos, una demanda por la precisión del rol de los dispositivos en la organización y disposición de los usos y de las apropiaciones. Sabemos que existe una distancia entre los mensajes y la recepción vinculada con las apropiaciones negociadas, oposicionales y dominantes que definió Hall (1979) y que luego redefinió Morley (1992). Lo cierto es que no hay infinitas formas de vincularse con los mensajes de los medios de comunicación y sus múltiples dispositivos sino direcciones hegemónicas de interpretación y producción consecuente.

Como sostienen Cabello y Moyano (2006) debemos enfocar los usos no solamente en recepción sino también en producción y entender a las TIC como dispositivo tecnológico-social que media positivamente prácticas de conectividad y de producción de conocimiento. Y es aquí donde radica la clave para pensar el lugar que ocupan las TIC y los dispositivos como tercera (gran) palabra clave para analizar nuestro corpus.

Redes sociales

El rol que las redes sociales han adquirido en los últimos años en el subcampo de estudio es impresionante. Decimos esto por la cantidad de publicaciones vinculadas con el tema pero, también, con la facilidad de acceso a los perfiles de usuarios para analizar sus interacciones, la construcción de sí frente a otros (en el sentido de Goffman, 1974) y las relaciones en red en términos generales. La proliferación de estudios se ve claramente en nuestro corpus en la forma de temas, variables y definiciones necesarias a la hora de pensar las "identidades virtuales" como las 
llama Passerini (2012). Para esta autora, la modificación de las categorías de tiempo y de espacio son las claves a la hora de analizar la relación a través de las TIC, que "nunca es faz a faz, siempre se haya mediatizada".

Arabito (2011) sostiene que en muy poco tiempo (nuevamente, el tiempo y el espacio como ejes para reflexionar sobre la contemporaneidad) se dieron importantes cambios: "[...] en un abrir y cerrar de ojos, nació un mundo de posibilidades del tipo DIY (Hacelo vos mismo), y de un momento a otro, quedó claro que la gente común y corriente podría crear material que cualquier otra persona con una conexión a Internet podría ver. Y esa fue la base de la Web 2.0 primero y posteriormente de las Redes Sociales. El prólogo de una experiencia que nos haría ver que la web no es solo una creación tecnológica sino también, profundamente cultural" (Arabito, 2011: 6). En esta línea propone como título "Nos encontramos en Facebook", como una nueva expresión propia de los tiempos que corren para abordar la interacción en época de redes sociales -en el sentido de Murolo (2011) en la comparación de dos subgrupos-.

Di Próspero (2009), en un avance de su tesis de maestría, sostiene que las formas de uso de Facebook y las prácticas sociales identificables, se ubican en un punto medio entre la "dominación" del dispositivo y la creatividad de la apropiación de las herramientas. Es decir, lejos de posturas deterministas, propone abordar los usos y las prácticas en torno a Facebook y reconoce "pequeñas tácticas" que hacen posible la creatividad (aunque no presenta un abordaje teórico-conceptual que lo sostenga como posibilidad).

El rol de las redes sociales (con especial eje en Facebook) puede explicarse por su novedad, por la potencia con la que se expandió y sumó usuarios pero, también, por la sencillez de su abordaje metodológico y analítico en la investigación. Es por ello que es nuestra última palabra clave. El tiempo dirá de qué manera se organiza la nueva bibliografía en torno a las redes y a las nuevas innovaciones. En lo que sigue, abordaremos las perspectivas y herramientas metodológicas que son, claramente, un tema fundamental a la hora de analizar el corpus. 


\section{Abordajes y estrategias metodológicas}

Como hemos mencionado, en el corpus analizado prevalece una concepción no determinista de las tecnologías y de los medios de comunicación, ya que se interpreta que los cambios culturales y tecnológicos se influyen mutuamente. El foco aparece puesto, entonces, en los procesos de recepción y en las mediaciones entre sujetos, medios y tecnologías. En líneas generales, la apropiación y el uso de TIC son entendidos como procesos que han transformado las formas de comunicación, las instancias de socialización, el funcionamiento de la economía, las prácticas y los consumos culturales.

En muchos casos los abordajes de estos procesos privilegian perspectivas comparativas sincrónicas, ya sea por generaciones, género, clases sociales y en diferentes escenarios (la escuela, la universidad, la vida cotidiana, el espacio público) (Cabello y Moyano, 2006; Albarello, 2011; Benítez Larghi et al. 2012; Aon y Zapata, 2011; Bianchi, López y Perera, 2008).

Otros trabajos dedican gran parte de su esfuerzo estableciendo un recorrido asincrónico de la evolución del objeto de estudio. Así, por ejemplo Arabito (2011) y Sanguinetti (2008), ofrecen una historización de los cambios tecnológicos analizando el desarrollo de nuevas formas de mediación y, en menor medida, el devenir de la experiencia de los usuarios. La primera sección del libro de Sanguinetti (2008) es elocuente de esto que decimos por cuanto logra contextualizar aquel desarrollo en el marco de procesos de transformación social más amplios. Allí el autor aborda las mutaciones societales desde la década de 1970 hasta la actualidad, poniendo énfasis en las transformaciones culturales y comunicacionales en el marco de los procesos de globalización y la particular inscripción de la Argentina en ellos.

Sanguinetti propone localizar las TIC en la cultura y es por ello que las mediaciones comunicativas deben ser pensadas desde una perspectiva distinta. "Ya no como medios de transmisión, intercambio, difusión, sino como formas y técnicas que modifican y transforman definitivamente nuestra "manera de estar juntos" (Sanguinetti, 2008: 23-25).

Este tipo de perspectiva y el modo de concepción de las TIC que venimos marcando a lo largo de todo el capítulo repercute en las formas de abordaje y estrategias metodológicas privilegiadas por los estudios del corpus. 
La primera parte del libro de Sanguinetti está basada en una extensa revisión bibliográfica. Tal como lo anticipa su autor al comienzo del libro. Los ejes planteados en esos capítulos giran en torno a los cambios en la socialidad a partir de la expansión de las TIC; el análisis de categorías como tecnología, técnica y tecnicidades; los modos en que las personas y grupos se apropian del espacio; la política micro y los nuevos sujetos que actúan en un contexto de globalización; los vínculos entre TIC y transformaciones del Estado. A medida que se avanza en las subsiguientes secciones, el libro va perdiendo relevancia para los fines de este capítulo, ya que la preocupación por los procesos de recepción, consumo y apropiación de las TIC se va diluyendo. La segunda parte analiza diferentes modos de vinculación entre la política, los medios de comunicación y las Tecnologías de Información y Comunicación, especialmente en la región de La Plata y Gran La Plata, mientras que la tercera parte está dedicada al análisis de la trama económica y política que opera detrás de los medios a partir del estudio de las normas y leyes que sostienen y legitiman a la Sociedad de la Información en la Argentina. Respecto a la segunda parte del libro, la metodología utilizada en los estudios de caso no resulta uniforme. Ante todo, hay que decir que solamente uno de los capítulos dedica una sección específica para detallar explícitamente la metodología utilizada mientras que en el resto debe deducirse de la presentación de los resultados. Por un lado, parece predominar el análisis de sitios web tanto de organizaciones sociales como de entes estatales o partidos políticos, cuestión que escapa a nuestro eje de análisis, ya que prácticamente no se estudia la recepción o la apropiación de esas plataformas. Solamente en algunos casos se utiliza la técnica de entrevistas. La tercera parte, decididamente fuera de nuestro alcance, está basada en el análisis documental de las normativas y leyes que sustentan el ordenamiento económico-político de los medios en la Argentina. Así, en líneas generales, el libro aparece dominado por el análisis bibliográfico y documental y estrategias cualitativas para llevar adelante el trabajo de campo. Sin embargo, sobre el final del libro, aparece un capítulo sobre usos y apropiaciones de TIC en el Gran La Plata donde se vuelcan -en clave de informe- los datos surgidos de una encuesta aplicada en la región distinguiendo accesos y prácticas más comunes según género, edad, nivel de escolarización y lugar de residencia. 
Este ejemplo final da cuenta de un factor recurrente en los estudios del corpus: un predominio de los estudios de corte cualitativo, preferentemente estudios de caso, pero mechado, varias veces, con la utilización de herramientas cuantitativas (Moscoloni y Castro Rojas, 2011; De la Torre y Vaillard, 2012; Bianchi, López y Perera 2008; Cabello y Moyano, 2006).

Sin embargo, cabe mencionar que la presencia de técnicas cuantitativas no implica necesariamente una estrategia de esa misma naturaleza. Más bien, el uso y recurso de estas herramientas nos permitiría conjeturar cierta preocupación por la construcción de conocimiento estable en línea con la identificación de las invariantes culturales del corpus (Geertz, 1987). En ese sentido, todos los textos explicitan una intención analítica propia de abordajes cualitativos. Uno de los mejores ejemplos al respecto es el exhaustivo trabajo de Monje (S/D) sobre los Centros Tecnológicos Comunitarios en Córdoba en el que la combinación de encuestas, entrevistas con informantes clave y con usuarios de estos espacios de acceso público a las TIC.

Dentro de las propuestas de combinación de técnicas pueden destacarse el ya citado caso de Albarello (2011) y el estudio De la Torre y Vaillard (2012). Por un lado, en su libro, Leer/navegar en Internet. Las nuevas formas de lectura en la computadora, Albarello interpreta los resultados de una investigación empírica, basada en un extenso y sistemático trabajo de campo que combina aproximaciones cuantitativas y cualitativas: una encuesta, una serie de entrevistas cualitativas; $y$, algo que comienza a ser una recurrencia en el sub-campo de estudios, un registro de las sesiones en Internet.

Por otro lado, el texto de De la Torre y Vaillard (2012) se orienta al análisis de la relación entre los jóvenes a nivel regional y las redes sociales más elegidas. En la persecución de este objetivo, merece subrayarse la doble metodología desplegada y, sobre todo, la escala de estudio, en tanto adquiere un alcance regional. Por un lado, el trabajo tiene una dimensión cualitativa y una cuantitativa. Por otro lado, la escala del trabajo es regional y los datos se han analizado considerando como unidad mínima (en la dimensión cuantitativa) los países (Argentina, Chile, Colombia, México y Uruguay). A lo largo del trabajo de campo, se realizaron grupos focales por país compuestos de estudiantes universitarios. Luego se entregó un cuestionario diseñado especialmente para analizar preferencias, frecuencias, prácticas y usos. Se realizaron 1269 encuestas en diferentes países a jóvenes universitarios (de 
establecimientos privados) entre 18 y 25 años a través del diseño de una muestra intencionada.

Finalmente, cabe indicar que buena parte del corpus está conformado por trabajos de corte ensayístico. Por lo tanto, estos textos no especifican la estrategia metodológica sino que simplemente se apoyan en datos de fuentes secundarias o en experiencias personales. Como por ejemplo el ya citado texto de Arabito (2011) en relación a los sentidos de Facebook o el de Contreras (2011) sobre los alcances y límites de la participación política a través del uso de TIC. Este último trabajo se propone visibilizar las posibilidades que ofrecen las nuevas tecnologías de comunicación para el ejercicio de acciones tendientes a producir potenciales cambios sociales, culturales y políticos. El eje de observación está puesto en las posibilidades de participación activa y espontánea, en la que los actores sociales, pueden convertirse a menudo en verdaderos "líderes de opinión", "ciberactivistas" o "propagandistas políticos" que desafían las barreras o "filtros" del orden establecido o lo "instituido" logrando de manera efectiva nuevos flujos de interacción, nuevas formas de acción estratégica y de relaciones sociales relevantes. Si bien este trabajo no se basa en el análisis empírico de un caso en particular, propone el concepto de ensamblaje socio-técnico (Bijker, 1995) para abordar metodológicamente el análisis y la interpretación de las interacciones de las comunidades en el ciberespacio. Para ello, parte de preguntarse cómo diferentes grupos que integran una determinada comunidad de usuarios sitúan al componente tecnológico como un lugar privilegiado para la participación, organización, producción e intercambio de ideas e información significativa. Mediante la descripción del caso WikiLeaks articula este interrogante con las posibilidades efectivas que ofrecen las tecnologías digitales a partir de la aparición de la Web 2.0.

Entendemos que el recurso al registro ensayístico presupone la legitimidad del objeto estudiado al tiempo en que evidencia la existencia de un sub-campo en conformación con pujante vigencia.

\section{Conclusiones}

El objetivo del capítulo se organiza, tal como lo dijimos al comienzo, en torno a la investigación realizada sobre las prácticas, las apropiaciones y el 
consumo de dispositivos y plataformas vinculadas con las TIC. A lo largo de sus diferentes secciones, intentamos delinear un camino de análisis y de sistematización que nos ha permitido, al menos temporalmente, componer trazados de recorridos y de decisiones teóricas, metodológicas y conceptuales que se vinculaban con una mirada sobre lo existente y con una necesaria definición del sub-campo en el que se insertan estas investigaciones. Si bien el corpus que abordamos no es representativo ni generalizable, nos ha permitido componer diversas "fotos" de momentos específicos del sub-campo en los últimos años (y en el segmento temporal sobre el que se despliega el PISAC).

Las características del sub-campo temático se ordenan, como mencionamos, en función del lugar privilegiado que las TIC tienen en la actualidad en la investigación en ciencias sociales. A su vez, identificamos muchos temas de relevancia que se vinculan con la ubicuidad de estas tecnologías en la vida cotidiana de los sujetos que se vinculan con ellas.

El gran abanico temático sobre el cual se ordenan las TIC como objeto de estudio en las ciencias sociales se vincula, en nuestra hipótesis de cierre, con el rol crucial que tienen las nuevas formas de comunicación en la actualidad. Los temas son amplios y se relacionan de diversas formas con las TIC: los usos educativos, la participación política, las transformaciones en los modos de lectura y en la forma de producción de contenidos, los juegos, la sociabilidad, la construcción de subjetividades y las nuevas formas de reproducción de las desigualdades. Este listado no desplaza otros usos ni privilegia los relevantes sino que ordena, en función del corpus de trabajo, la composición del sub-campo en una orientación específica en un momento histórico, cultural, económico y político determinado.

Encontramos, como invariante, una preocupación por la relación entre los sujetos y las TIC en un muy amplio sentido y, complementariamente, una reflexión sobre la brecha digital que se abre en función del acceso, de las pragmáticas de uso y de apropiación de las TIC.

Mencionamos, al comienzo del capítulo, tres hipótesis que organizarían el texto: en primer lugar, la relación de las investigaciones sobre TIC con la caducidad de los objetos analizados; en segundo lugar, conjeturamos que el aumento de investigaciones sobre las TIC podría vincularse con un acceso sencillo a las formas de uso de las plataformas y redes sociales; y, en tercer lugar, mencionamos la "competencia desleal" entre las TIC 
y las otras formas de comunicación (analógicas y digitales) en y sobre las cuales se puede investigar.

Las tres hipótesis fueron sostenidas a lo largo del trabajo con ejemplos, discusiones y debates y fue posible encontrar cada una de ellas en las formas en las que las investigaciones se construían metodológicamente pero, también, en la manera en la que los objetos de estudio se desplegaban para su análisis a lo largo de ponencias, libros, capítulos y artículos.

Es por ello que al pensar en el futuro cercano del sub-campo aparece una constatación casi indiscutible: el terreno sobre el que se asientan las TIC está en constante modificación y la investigación construye repertorios de referencias temporales sobre los dispositivos, usos y apropiaciones posibles (e imposibles) en un momento específico del presente. Este mismo capítulo contiene en sí el gen de su propia caducidad pero contiene, a su vez, un potencial sintetizador sobre lo existente hasta el momento de escritura. El creciente interés en analizar la relación entre las TIC, los sujetos sociales y las prácticas no puede sino entenderse como intento sostenido de comprender las maneras en las que vivir en la sociedad contemporánea modifica de manera sustancial nuestra relación con el mundo y con las mediaciones (y dispositivos) con las que nos vinculamos (intencionalmente o no).

A su vez, la relación de las TIC con los medios de comunicación "tradicionales" no es menor: la preocupación constante de la radio y de la televisión por analizar, presentar casos (en general negativos) de mal uso o de preocupaciones sobre las TIC las ubica en un espacio de articulación de los miedos, las fantasías y las amenazas que pueden aparecer vinculadas a ellas. Es aquí donde encontramos, una vez más, el potencial del sub-campo en la definición de sus temas y en sus intentos de dar respuesta a las "demandas" sociales (representadas por los otros medios de comunicación como cristalizaciones del sentido común).

En síntesis, el sub-campo que componen las TIC es no solo muy productivo sino que está sometido a los cambios y avances de los dispositivos y formas de conexión disponibles. La pregunta que nos queda, para cerrar este capítulo, se vincula con las herramientas metodológicas: ‘acaso el sub-campo demanda una adecuación de las herramientas de investigación para seguir su ritmo o es que la investigación no puede avanzar en los tiempos pautados por las apariciones y desarrollos del mercado? La respuesta a este interrogante se encuentra no solo en el campo académico- 
científico sino, también, en el comercial y en las formas que adquiere el ecosistema comunicativo en el que vivimos.

\section{Referencias bibliográficas}

Becerra, M. (2003), Sociedad de la Información: proyecto, convergencia, divergencia, Norma, Buenos Aires.

BijKer, W. (1995), Of Bicycles, Bakelites, and Bulbs: Toward a Theory of Sociotechnical Change, MIT Press, Cambridge.

Cabello, R. (2006), Yo con la computadora no tengo nada que ver. Un estudio de las relaciones entre los maestros y las tecnologías informáticas en la enseñanza de 2006, UNGS, Prometeo.

Fidler, R. (1998), Mediamorfosis. Comprender los nuevos medios, Granica, Buenos Aires.

Finquelevich, S. y Prince, A. (2007), El (involuntario) rol social de los cibercafés, Dunken, Buenos Aires.

Fuichy, P. (2013), «Rendre visible l'information. Une analyse sociotechnique du traitement des donnés», Reseaux, No 178-179, pp. 55-89.

Geertz, C. (1987), La interpretación de las culturas, Gedisa, Barcelona.

Goffman, E. (1974), Frame Analysis, Northeastern University Press, Boston.

Gubern, R. (2000), El eros electrónico, Taurus, Madrid.

Hall, S. (1979), "Encoding/Decoding.", en Hall, S. y otros, Culture, Media, Languaje. Working Papers in Cultural Studies, 1972-79, Hutchinson, Londres, pp. 128-138.

Mastrini, G., Loreti, D. y Baranchuk, M. (2007), Participación y democracia en la sociedad de la información, Prometeo, Buenos Aires.

Morley, D. (1992), Television, Audiences and Cultural Studies, Routhledge, Londres y Nueva York.

Quintar, A., Calello, T y Aprea, G. (comps.) (2007), Los usos de las TICS. Una mirada multidimensional, Prometeo y UNGS, Buenos Aires.

Varela, M. (2005), La televisión criolla. Desde sus inicios hasta la llegada del hombre a la luna 1951-1969, Edhasa, Buenos Aires.

Williams, R. (1974), Television. Technology and Cultural Form, Fontana, Hannover y Londres. 


\section{Corpus analizado}

Albarello, F. (2011), Leer/navegar en Internet. Las nuevas formas de lectura en la computadora, La Crujía, Buenos Aires.

Alzina, P., Benyo, J. y Durán Prieto, J. (2011), "Usos y apropiaciones de las TIC en movimientos territoriales y asambleas barriales", Avatares de la Comunicación y la Cultura, $N^{\circ} 2$, pp. 1-18.

Andrés, G. (2012), "Prácticas culturales en red: reflexión sobre la participación y producción de los internautas", Poliantea, N VII (14), pp. 97-110.

Aon, L. y Zapata, N. (2011), "Jóvenes y consumos culturales. Una mirada sobre la recepción tecnológica y audiovisual en estudiantes de Comunicación Social", Question, $N^{\circ} 1$ (30). Recuperado de <http://perio.unlp.edu.ar/ojs/index.php/question/article/view/1051/1048>. Consulta: 27 de octubre de 2015.

Arabito, J. (2011), “¿Nos encontramos en Facebook? Las redes sociales construyen a la Web 2.0 como espacio de interacción social en metamorfosis", en Villafañe, A. y Adad, L. (comps.), Antropología y crisis global: desafios para una interpretación desde el sur, Departamento de Antropología y NURES, FACSO, Olavarría.

Benítez Larghi, S. et al. (2012), "La apropiación del acceso a computadoras e Internet por parte de jóvenes de sectores populares urbanos en la Argentina”, en Proenza, F. (ed.), Tecnología y Cambio Social. El impacto del acceso público a las computadoras e Internet en Argentina, Chile y Perú, IDRC e Instituto de Estudios Peruanos, Lima.

Bianchi, M., López, G. y Perera, V. (2008), "Usos de Internet en adolescentes de Chubut", en Jornadas Nacionales de Investigadores en Comunicación, Rosario.

BonaccI, J.M. (2008), “Tejiendo el rock en la red: usos y prácticas de webmasters roqueros”, en Urresti, M. (ed.), Ciberculturas juveniles, La Crujía, Buenos Aires.

Bouille, J. (2008), “Cibercafés o la nueva esquina. Usos y apropiaciones de Internet en jóvenes de sectores populares urbanos", en URRESTI, M. (comp.), Ciberculturas juveniles, La Crujía, Buenos Aires.

Cabello, R. y Moyano, R. (2006), "Hábitos de consumo de juegos en red en zonas periurbanas. Estudio de caso en Área Metropolitana de Buenos Aires, Razón y Palabra [en línea], No 11, diciembre-enero), Recuperado de: <http:// 
www.redalyc.org/articulo.oa?id=199520736022> [Consulta: 27 de octubre de 2015].

Contreras, L. (2011), "Las posibilidades de participación política mediante las tics: Visibilidad en un nuevo espacio público ¿Ingenuidades o certezas?”, en Bianchi, M. y Sandoval, L. (eds.), Jornadas Patagónicas de estudios sociales sobre Internet y tecnologías de la comunicación, Universidad Nacional de la Patagonia San Juan Bosco, Comodoro Rivadavia.

Cornejo, M. y TApia, M. (2011), "Redes sociales y relaciones interpersonales en Internet”, Fundamentos en humanidades, N XII (2), pp. 219-229.

De la Torre, L. y Vaillard, L. (2012), “¿Cómo usan las redes sociales los jóvenes de Latinoamérica?", Ecos de la Comunicación, No 5 (5). Recuperado de http://bibliotecadigital.uca.edu.ar/repositorio/revistas/como-usan-redes-sociales-jovenes.pdf [Consulta: 27 de octubre de 2015].

Di Próspero, C. (2009), “Tecnologías infocomunicacionales y reconfiguraciones de los espacios de significación en la vida cotidiana. Usos de la red social Facebook en jóvenes de la Ciudad de Buenos Aires", en V Jornadas de jóvenes investigadores, Instituto de Investigaciones Gino Germani, Universidad de Buenos Aires, Buenos Aires.

Fernández Jeansalle, Julia (2008), "Doble clic. Internet y jóvenes de clase media en la ciudad de Buenos Aires", en Urresti, Marcelo (ed.), Ciberculturas juveniles, La Crujía, Buenos Aires.

Goszczynski, Laura (2008), "Hacia los usos adolescentes del fotolog: vía ¡libre? para la presentación de sí”, en URresti, M. (ed.), Ciberculturas juveniles, La Crujía, Buenos Aires.

JАСОВо, I. (2011), "Historietas 2.0: nuevas formas de producir y consumir historietas", en Bianchi, M. y Sandoval, L. (eds.), en Jornadas Patagónicas de estudios sociales sobre Internet y tecnologías de la comunicación, Universidad Nacional de la Patagonia San Juan Bosco, Comodoro Rivadavia.

Maestri, M. (2011), "Las audiencias digitales: convergencias y prácticas", en Valdettaro, S. (comp.), Interfaces y pantallas: análisis de dispositivos de comunicación, UNR Editora, Rosario.

Marchetti, V., Cantor, F., Musa, C., Poletti, M. y Vergini, M. (2008), “El impacto de las tecnologías de pantalla en personas con discapacidad", La Trama de la Comunicación, No 13, pp. 37-50.

Monje, D. (s/d), Lo maravilloso moderno. Resignificación y derivas del GII en la República Argentina: estudio del caso de los Centros Tecnológicos Comunitarios, 
Maestría en Comunicación y Cultura Contemporánea-CEA, UNC, Córdoba.

Moscoloni, N. y Castro Rojas, S. (2011), "Consumos de dispositivos tecnológicos: usos de pantallas en ingresantes a la universidad", en Valdettaro, S. (comp.), Interfaces y pantallas: análisis de dispositivos de comunicación, UNR Editora, Rosario.

Murolo, N. (2011), "Videojuegos. Usos y prácticas comunicacionales", en Bianchi, Marta Pilar y Sandoval, Luis Ricardo (eds.), en Jornadas Patagónicas de estudios sociales sobre Internet y tecnologías de la comunicación, Universidad Nacional de la Patagonia San Juan Bosco, Comodoro Rivadavia.

Pahor, M. (2008), Conectad@s. Cómo usan Internet los jóvenes de clase alta en Buenos Aires, en Urresti, M. (ed.), Ciberculturas juveniles, La Crujía, Buenos Aires.

Passerini, A. (2012), "Relaciones en la Red: sus vicisitudes y sus quejas”, en VII Jornadas de Sociología de la Universidad Nacional de La Plata. "Argentina en el escenario latinoamericano actual: debates desde las ciencias sociales", Facultad de Humanidades y Ciencias de la Educación, La Plata.

PAz, M. (2012), “Usos educativos juveniles de las TICs”, X ENACOM, Posadas.

Remondino, G. (2012), "Blog y redes sociales: un análisis desde las tecnologías de la gubernamentalidad y el género", Athenea Digital,Nº 12 (3), UOC, Barcelona, pp. 1-24.

Sandoval, L. (2008), ¿El fin de los medios? Politicas, apropiaciones y usos de las TIC, EPC Medios, La Plata.

SanguinetTi, L.P. (ed.) (2008), ¿El fin de los medios? Políticas, apropiaciones y usos de las TICs, Ediciones de Periodismo y Comunicación, La Plata.

Urresti, M. (2008), Ciberculturas juveniles, La Crujía, Buenos Aires.

Vannini, P. (2008), "La comunidad del siglo XXI. Grupos de usuarios de software libre", en Urresti, M. (ed.), Ciberculturas juveniles, La Crujía, Buenos Aires. 